\title{
船-
}

\section{La influencia jurídica en los Trabajos de Fin de Grado en Trabajo Social en la Universidad de Salamanca}

\author{
María SaAvedra GutiérRez \\ Departamento de Derecho del Trabajo y Trabajo Social \\ UNIVERSIDAD DE SALAMANCA \\ masaagu@usal.es
}

Resumen: Durante los últimos años, los Trabajos de Fin de Grado (TFG) han emergido como una importante fuente de información e investigación en el campo académico del Trabajo Social. En esta comunicación se analiza la influencia del Derecho y las estrategias metodológicas empleadas por el alumnado del grado en Trabajo Social de la Universidad de Salamanca durante los años 201218. Se han estudiado un total de 781 trabajos. Como conclusiones principales podemos destacar la hegemonía de la metodología cuantitativa, la prevalencia de temáticas contextualizadas en el marco del Derecho, y en el ámbito del Derecho Público General como temática recurrente.

Palabras Claves: trabajo fin de grado, derecho, trabajo social. 


\title{
The influence of law in Final Degree Projects in Social Work at the University of Salamanca
}

\begin{abstract}
Throughout the past few years, Final Degree Projects, or FDP (known as Trabajos de Fin de Grado or TFG in Spanish), have emerged as an important source of information and research within the academic field pertaining to Social Work. In this paper, the influence of Law is analysed, along with the methodological strategies used by undergraduates studying for a degree in Social Work at the University of Salamanca between 2012 and 2018. A total of 781 papers were analysed altogether. As our main conclusions, we can highlight the hegemony of the quantitative approach, the prevalence of contextualised issues within the framework of Law, and the field of General Public Law as a recurring theme.
\end{abstract}

Keywords: final degree project, law, social work. 


\section{La influencia jurídica en los Trabajos de Fin de Grado en Trabajo Social en la Universidad de Salamanca}

\section{INTRODUCCIÓN}

El título de Grado en Trabajo Social comienza a impartirse en el curso 2009-10 en la Universidad de Salamanca, una vez superado el proceso de verificación, y sustituye al de Diplomado en Trabajo Social.

En febrero de 2012 comenzó a impartirse, la primera edición del Curso de Adaptación al Grado en Trabajo Social, con el fin de que los Diplomados en Trabajo Social, titulados de la anterior ordenación, obtengan, tras superarlo, el título de Graduado en Trabajo Social (actualmente no se ofertan plazas para el citado curso de adaptación).

Entre las novedades incorporadas a las titulaciones de Grado, se encuentra la realización y defensa de un trabajo final en el que se acreditan los conocimientos y destrezas adquiridas a lo largo de la titulación. En esencia el TFG corresponde a un trabajo autónomo y personal que cada estudiante realizará bajo la orientación de un profesor. El trabajo permitirá al estudiante mostrar de forma integrada los contenidos formativos recibidos y las competencias adquiridas asociadas al título de Grado. 
El TFG podrá adoptar alguna de las siguientes modalidades:

a) Trabajo de investigación.

b) Trabajo teórico y revisión bibliográfica.

c) Proyecto de intervención y/o trabajos de sistematización profesional.

\section{Trabajo de investigación}

En un trabajo de investigación social se busca explicar, describir, explorar, comprender... la realidad social que va a ser objeto de estudio. Se parte de una pregunta formulada con claridad, a la que se busca dar respuesta utilizando, para ello, el método científico. A continuación se presenta un esquema orientativo sobre la estructura y el contenido que debe tener esta modalidad de TFG:

- Resumen y palabras clave en castellano e inglés.

- Introducción, justificación y delimitación del objeto de investigación

- Fundamentos teóricos y revisión bibliográfica (un mínimo de 15 referencias).

- Diseño metodológico, técnicas y fuentes de investigación.

- Análisis, presentación y discusión de los resultados

- Conclusiones y aportaciones teóricas y metodológicas.

- Bibliografía

- Anexos (si los hubiera).

\section{Trabajo teórico y revisión bibliográfica}

Como segunda modalidad, el alumno puede optar en su TFG por la elaboración de referentes/marcos teóricos y/o normativos sobre contenidos y competencias de interés profesional/investigador. Este tipo de trabajos pueden entenderse como un conjunto de informaciones precisas sobre un tema determinado que necesita de un proceso de búsqueda e interpretación exhaustiva de las diversas fuentes documentales existentes. El trabajo podrá ser de tipo panorámico, cuando se presenten estudios de síntesis o exposición sobre un tema, o de tipo monográfico, 
cuando se aborde de forma más precisa el objeto de estudio. La estructura y contenido que debería incluir este tipo de TFG sería la siguiente:

- Resumen y palabras clave en castellano e inglés.

- Introducción y justificación del objeto de estudio.

- Revisión e investigación bibliográfica (un mínimo de 20 referencias).

- Discusión teórica y formulación de interrogantes.

- Conclusiones.

- Bibliografía.

- Anexos (si los hubiera).

\section{Proyecto de intervención y/o trabajos de sistematización profesional}

El Proyecto de Intervención puede entenderse como un conjunto articulado de actividades que se diseñan para conseguir unos objetivos dentro de los límites de un presupuesto y un período de tiempo establecidos. Se orienta a la mejora, la solución o la satisfacción de necesidades, problemas o situaciones sociales. El diseño de este tipo de proyectos implica una reflexión y una toma de conciencia sobre las necesidades existentes (diagnóstico social), y la elaboración de un diseño científico, cuya aplicación sirva para transformar y mejorar la realidad social con la que se quiere trabajar.

A continuación se presenta un esquema orientativo sobre la estructura y el contenido que debe tener esta modalidad de TFG

- Resumen y palabras clave en castellano e inglés.

- Introducción y justificación: relación con objetivos y competencias del Grado, oportunidad y conveniencia del proyecto y/o de la sistematización.

- En proyectos de intervención: fundamentos teóricos, revisión bibliográfica (un mínimo de 15 referencias), objetivos, destinatarios, diseño metodológico, acciones y medidas, cronograma, sistemas de evaluación, recursos humanos, económicos y técnicos, resultados y beneficios esperados. 
- En trabajos de sistematización profesional: recopilación y presentación de datos e información, análisis e interpretación, relevancia teórica y profesional, etc.

- Bibliografía.

- Anexos (si los hubiera)

\section{MÉTODO}

El objetivo general pretende, por tanto, analizar las temáticas y opciones metodológicas desarrolladas en el Grado en Trabajo Social en el periodo que va del curso 2011-12 al 2017-18. Se emplea para ello una metodología cuantitativa basada en el uso de datos secundarios, utilizando como fuente los trabajos depositados en la Secretaria de la Facultad de Ciencias sociales durante el periodo indicado.

Durante los cuatro años en los que convivieron el grado y el curso de adaptación se defendieron 781, de los cuáles 259 corresponden al curso de adaptación al grado (2011-2015) y 522 al grado propiamente dicho (2012-2018). Analizaremos qué y cómo investigan los alumnos de Trabajo Social.

Examinaremos, por un lado, la modalidad más frecuente de TFG elegida por los alumnos del Grado de Trabajo Social de Salamanca y por el otro, la presencia del derecho en los mismos. 


\section{RESULTADOS}

De la totalidad de los trabajos realizados durante los años 2012-2018, la opción elegida mayoritariamente en un 44\% por el alumnado es la modalidad c, correspondiente a la realización de un proyecto de intervención; en un $43 \%$ es la modalidad b (Trabajo teórico y revisión bibliográfica) y en un 13\% la modalidad a (proyecto de investigación). Quedando gráficamente de la siguiente manera:

\section{Gráfico 1 \\ Modalidad TFG en Trabajo Social 2012-2018}

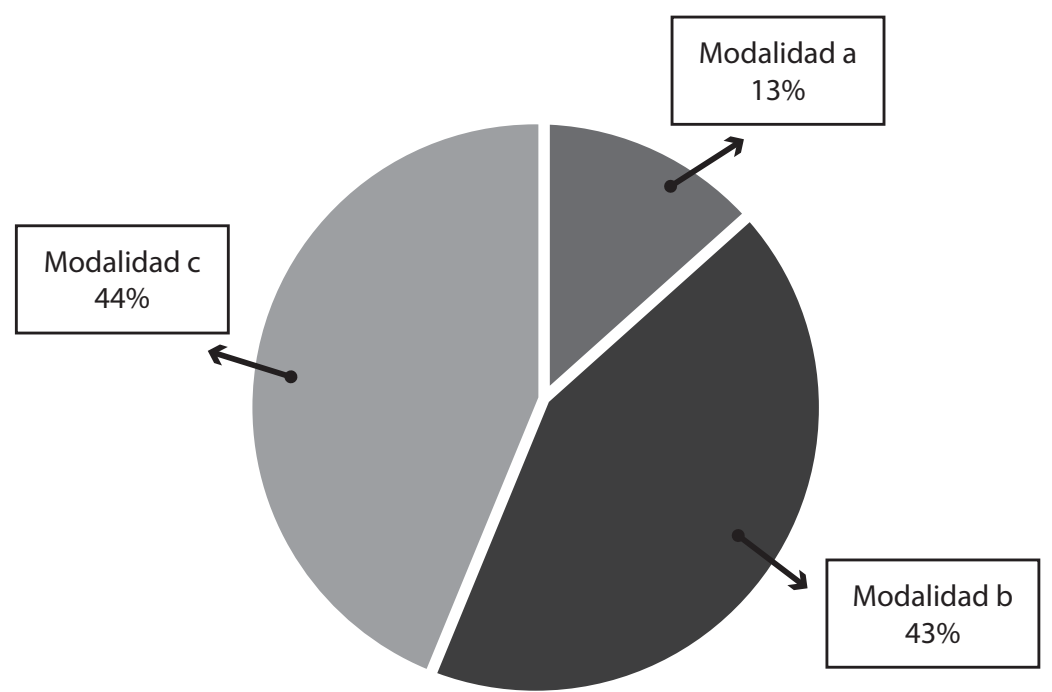

Fuente. Elaboración propia a partir de datos de la Secretaría de la Facultad de Ciencias Sociales de la USAL. 
Si examinamos por un lado, los cursos de adaptación al grado y por el otro, los de grado propiamente dicho, con la finalidad de hacer el estudio lo más exhaustivo posible, el resultado obtenido es el siguiente: de la totalidad de los 259 trabajos presentados durante los años 2011-2015, 45 corresponden a la modalidad a; 96 a la b y 116 a la modalidad c; quedando expresados descriptivamente del siguiente forma:

\section{Gráfica 2}

Modalidad de TFG cursos de adaptación al grado en Trabajo Social

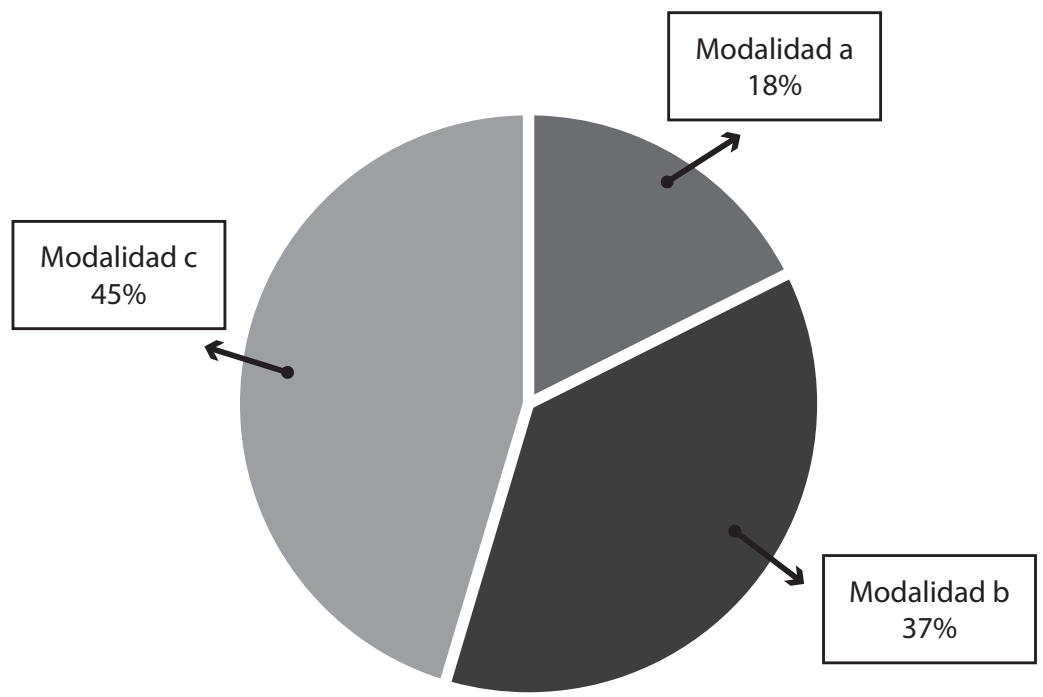

Fuente. Elaboración propia a partir de datos de la Secretaría de la Facultad de Ciencias Sociales de la USAL. 
A continuación, revisamos los trabajos realizados por año en cada una de las modalidades recogidas en la normativa específica del Título de Grado para la realización de los TFG: Modalidad a (Trabajo de investigación), Modalidad b (Trabajo teórico y revisión bibliográfica) y Modalidad c (proyecto de intervención).

\section{Gráfica 3}

\section{Cursos de adaptación al grado. Modalidad TFG por año}

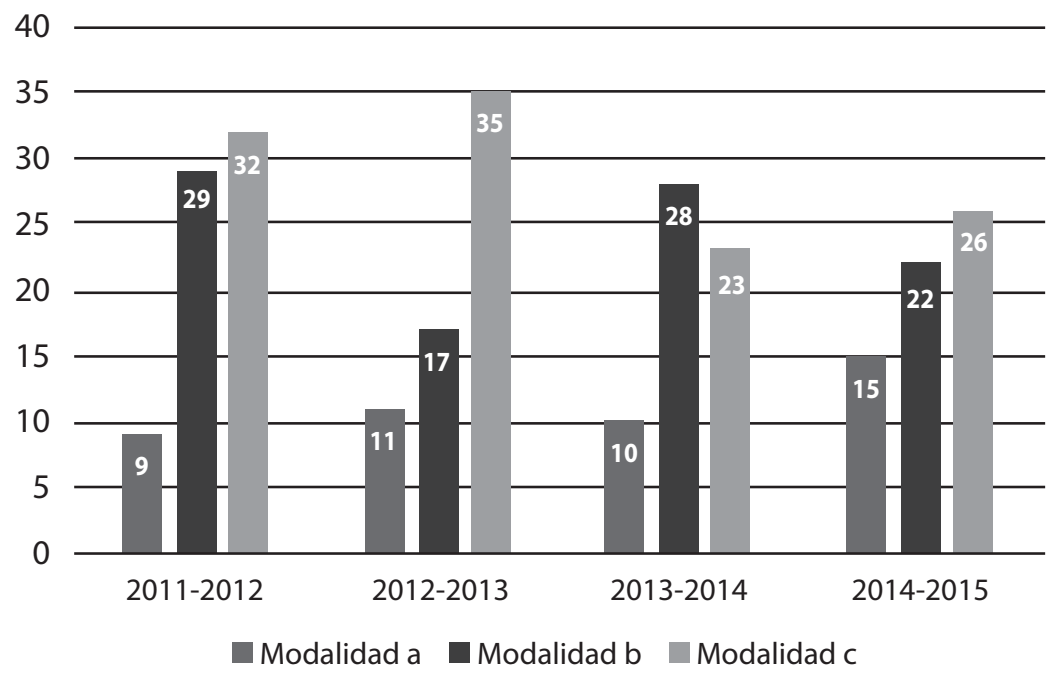

Fuente. Elaboración propia a partir de datos de la Secretaría de la Facultad de Ciencias Sociales de la USAL. 
En el Grado de Trabajo Social durante los años 2012-2018, se presentaron 522 Trabajos, de los cuales, 58 pertenecen a la modalidad a; 239 a la modalidad b y 225 a la modalidad c, siendo la opción elegida mayoritariamente por los alumnos del grado la modalidad b (correspondiente con el trabajo teórico y revisión bibliográfica).

\section{Grafico 4 \\ Modalidad TFG en el Grado en Trabajo Social}

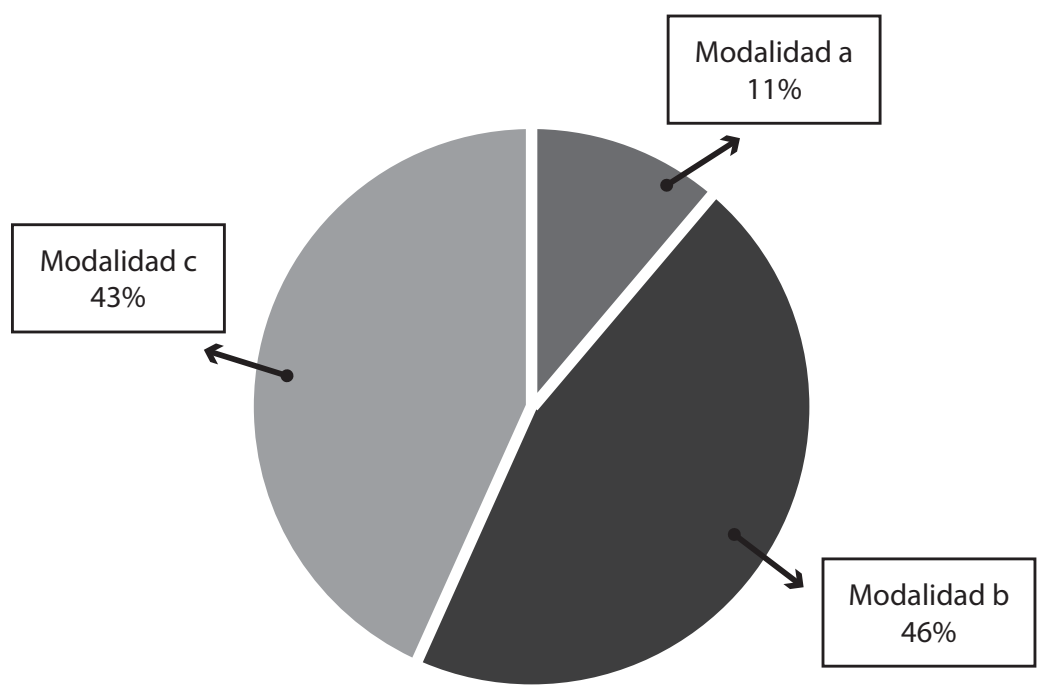

Fuente: elaboración propia a partir de datos de la Secretaría de la Facultad de Ciencias Sociales de la USAL.

A diferencia de lo ocurrido con los alumnos del curso de adaptación para la obtención del Grado en Trabajo Social que optaron por la realización de un proyecto de intervención como la modalidad elegida por la mayoría (modalidad c). 
Seguidamente, examinamos las diferentes modalidades elegidas por los alumnos a la hora de realizar su trabajo de fin de grado mostrando año por año las preferencias del alumnado.

\section{Gráfica 5}

Modalidad TFG en el Grado de Trabajo Social por año

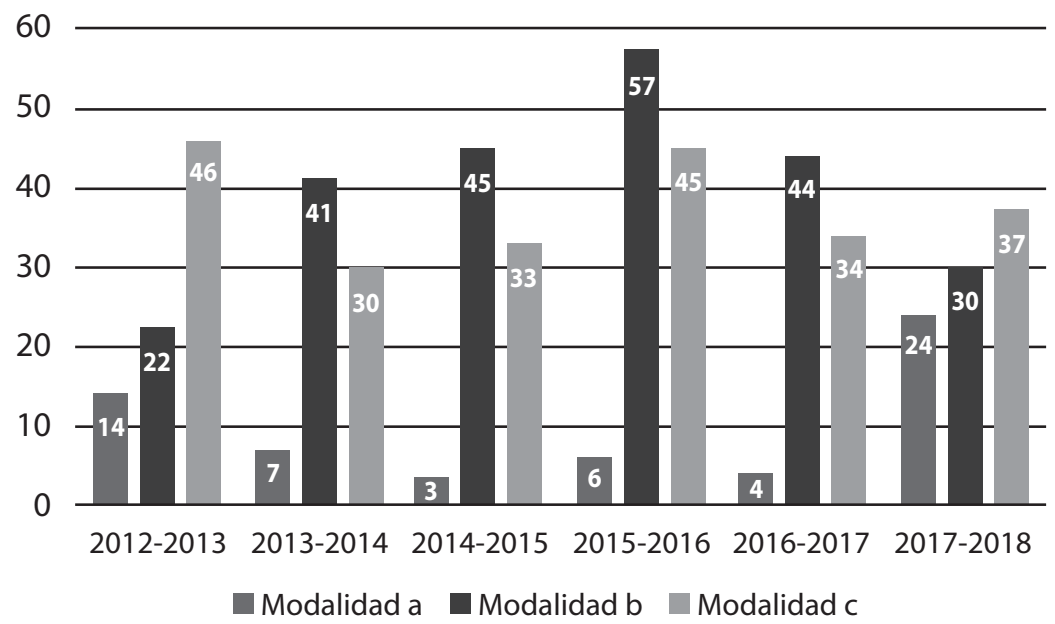

Fuente: elaboración propia a partir de datos de la Secretaría de la Facultad de Ciencias Sociales de la USAL. 
Hasta aquí hemos visto qué modalidad de TFG era la elegida por los alumnos del Grado en Trabajo Social, a continuación vamos a ver qué influencia tiene el Derecho en ellos.

Vamos a utilizar tres ítems: aquellos trabajos en los que no hay presencia alguna de normativa legal o jurídica o que su presencia es escasa/mínima (denominamos nada o poco), aquellos en los que se desarrolla el marco normativo o jurídico de la materia objeto de Trabajo (denominamos bastante) y en los que el trabajo se basa en algún ámbito del Derecho (mucho).

Siguiendo la línea establecida con la modalidad de los trabajos, de la totalidad de los mismos, durante los años 2012-2018: 406 no contienen referencia alguna a normativa legal o jurídica, 276 si tienen reflejo de la normativa legal-jurídica y 92 son trabajos realizados en alguna materia /rama concreta de Derecho. De manera descriptiva quedaría de la siguiente forma representado:

\section{Gráfico 6}

\section{Contenido legal-jurídico de los TFGs en Trabajo Social 2012-2018}

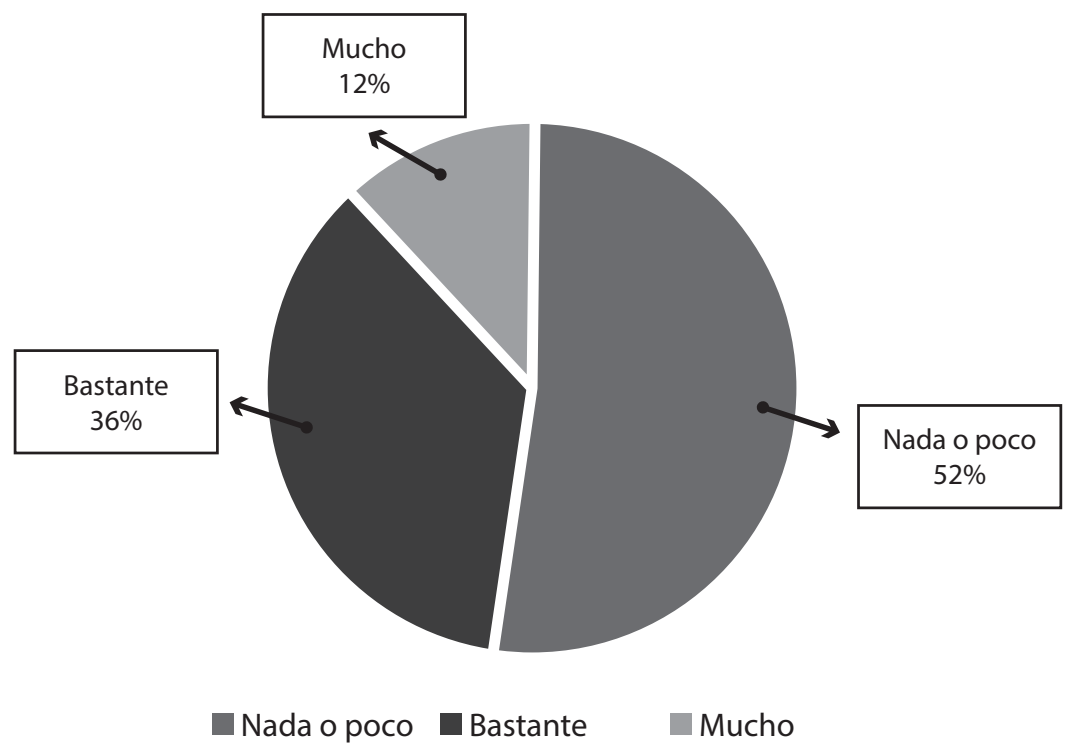

Fuente. Elaboración propia a partir de datos de la Secretaría de la Facultad de Ciencias Sociales de la USAL 
De los 259 Trabajos presentados por los alumnos durante el período comprendido entre los años 2012 y 2015, 84 de ellos contenían normativa jurídica-legal (36\%) bajo diferentes concepciones tales como: marco legal, marco legislativo, marco normativo, antecedentes, evolución y/o reformas legislativas, fundamentación jurídica, mecanismos jurídicos de protección, normativa supranacional y/o nacional, legislación significativa del tema...

\section{Gráfico 7 \\ Derecho en los TFG Curso de adaptación al grado (2012-2015)}

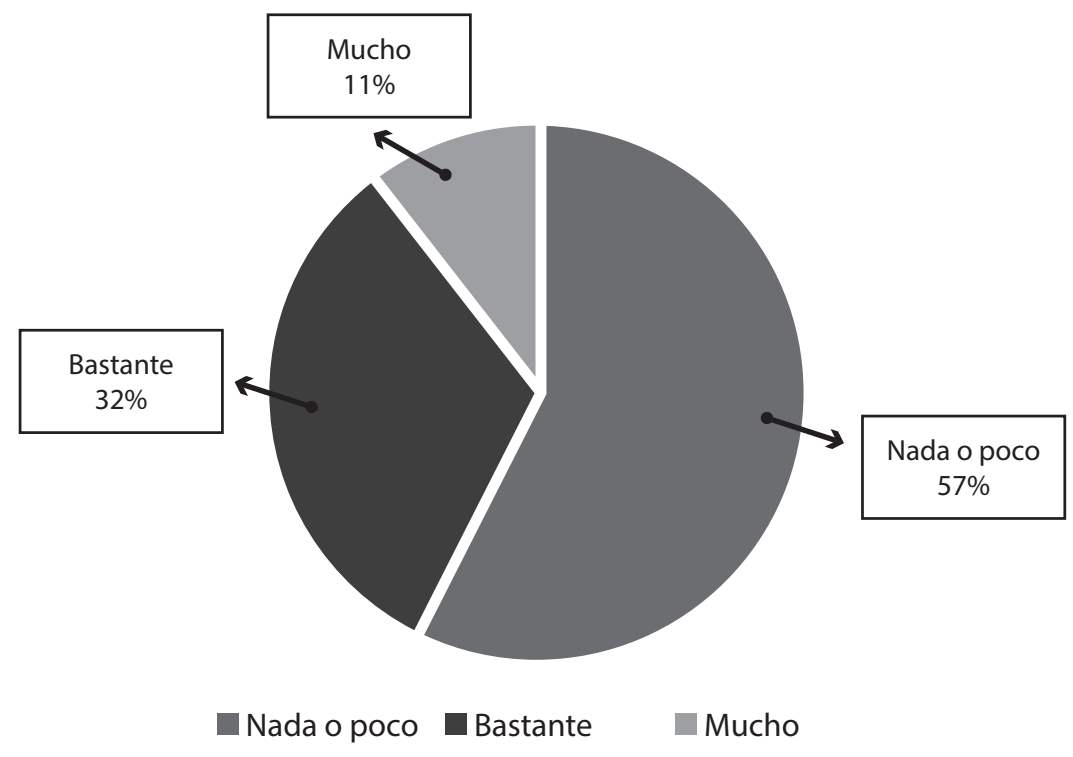

Fuente. Elaboración propia a partir de datos de la Secretaría de la Facultad de Ciencias Sociales de la USAL

Y un 12\% (concretamente 27) se realizan sobre algún ámbito concreto del Derecho, destacamos entre los temas elegidos por los alumnos los siguientes:

- Procedimiento administrativo; el reglamento administrativo.

- Régimen jurídico de las prestaciones; prestaciones no contributivas de la seguridad social; evolución 
normativa del ordenamiento jurídico español en materia de viudedad y/orfandad; el sistema de protección de la seguridad social: pensiones.

- Sistema penitenciario español; permisos penitenciarios.

- Asesoramiento legal sobre la violencia de género.

A continuación, lo vemos representado gráficamente por año (curso académico):

\section{Gráfico 8 \\ Derecho en los TFG curso de adaptación grado Trabajo Social por año}

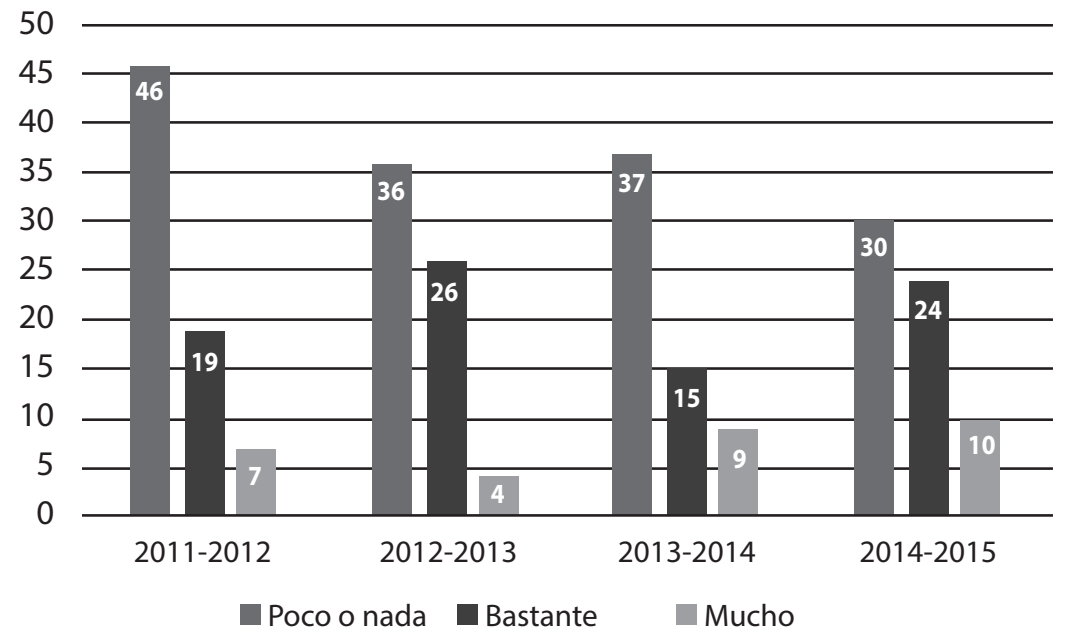

Fuente. Elaboración propia a partir de datos de la Secretaría de la Facultad de Ciencias Sociales de la USAL 
Durante el curso 2012-2013 se gradúa la primera promoción del Grado en Trabajo Social en Salamanca, desde estos años hasta el 2018 se presentan un total de 514 TFGs, de los cuáles 192 (37\%) muestran en su contenido reflejo expreso en algún momento a la normativa legal vigente; marco normativo-institucional; al marco jurídico; reformas legislativas; análisis de la normativa internacional, nacional, autonómica y local de una determinada materia; marco legislativo; medidas legales; base legal... Y 65 (13\%) trabajos son realizados en algún ámbito concreto del Derecho.

\section{Gráfico 9 \\ Derecho en los TFG Grado Trabajo Social}

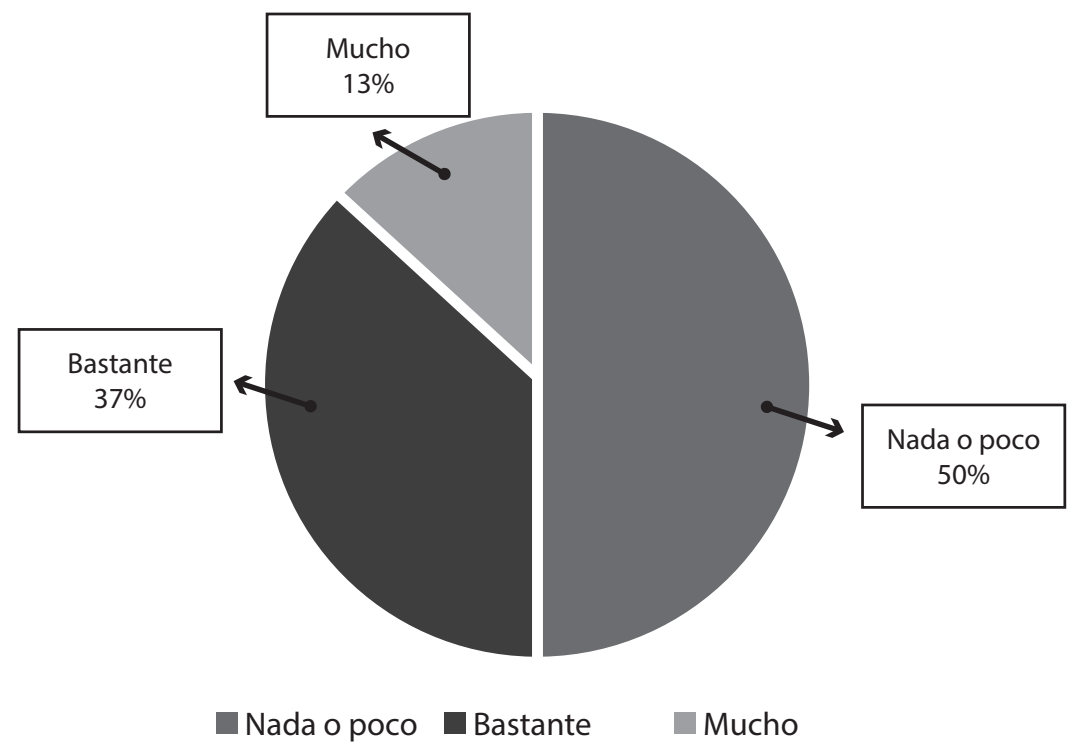

Fuente. Elaboración propia a partir de datos de la Secretaría de la Facultad de Ciencias Sociales de la USAL 
Seguidamente procedemos a examinar año por año el influjo de la normativa jurídico-legal en los TFGs realizados por los alumnos de Grado de Trabajo Social en la Universidad de Salamanca durante los años 2013-2018.

\section{Gráfico 10 \\ Derecho en los TFGs Grado Trabajo Social por año.}

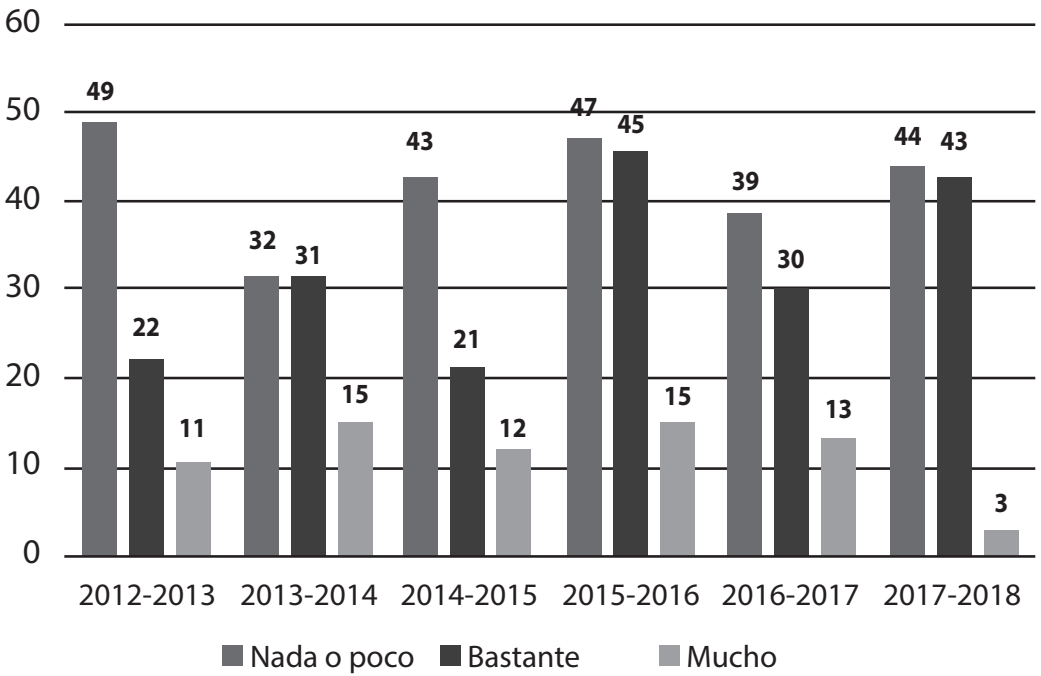

Fuente. Elaboración propia a partir de datos de la Secretaría de la Facultad de Ciencias Sociales de la USAL.

Durante estos años los alumnos que eligieron como tema de su Trabajo algún ámbito del Derecho fueron un total de 65, optando por los siguientes:

- Sistema de la Seguridad Social: sistema de pensiones español, pensiones no contributivas, prestaciones por desempleo y pensiones de incapacidad permanente, incapacidad temporal.

- Sistema penitenciario: régimen penitenciario para las personas con discapacidad intelectual, reinserción socio-laboral para mujeres y/o extranjeros, ámbito penitenciario para personas con enfermedad mental, reeducación de los hombres con condena 
por delito contra la violencia de género, mediación penitenciaria.

- Delincuencia juvenil y Ley Orgánica de Responsabilidad del Menor, mediación penal juvenil, mediación familiar, Justicia restaurativa y mediación.

- Análisis legislativo de la protección a la infancia, marco jurídico de la infancia.

- Protección de las personas con discapacidad en el ámbito civil, incapacidad y tutela de las personas con discapacidad intelectual, la incapacitación judicial.

- Procedimiento administrativo, potestad reglamentaria administrativa.

- Revisión de la legislación española sobre el aborto.

\section{CONCLUSIONES}

Los Trabajos de Fin de Grado se han convertido en una fuente importante de información e investigación para y sobre el Trabajo Social.

Tras el análisis realizado durante el período comprendido entre los años 2012-2018, podemos resaltar como principales conclusiones la hegemonía de la metodología cuantitativa, la prevalencia de temáticas contextualizadas en el marco del Derecho, y en concreto en el ámbito del Derecho Público General, el derecho penal y/o penitenciario y el derecho de la seguridad social, como temáticas recurrentes.

La modalidad de Trabajo de Fin de Grado preferida por la mayoría del alumnado del Grado de Trabajo Social de la Universidad de Salamanca es la realización de un proyecto de intervención, tanto en los cursos de adaptación al Grado (2011-2015) como en los del Grado propiamente dicho (2012-2018). De la totalidad de los Trabajos Fin de Grado presentados durante estos últimos años casi un tercio de ellos contienen referencia alguna sobre un ámbito concreto de nuestro Derecho Público General, materias tales como: delincuencia juvenil, sistema penitenciario y reinserción socio-laboral y/o reeducación, prestaciones económicas y sistema de pensiones español... 


\section{BIBLIOGRAFÍA}

Trabajos Fin de Grado en Trabajo Social depositados en la Secretaría de la Facultad de Ciencias Sociales de la Universidad de Salamanca.

Normativa específica del Trabajo de Fin de Grado en el Grado de Trabajo Social de la Universidad de Salamanca (aprobada en la Junta de la Facultad de Ciencias Sociales en sesión de 1 de junio de 2017) 